\title{
A EXPLORAÇÃO DE GÁS NATURAL EM MEXILHÃO: ANÁLISE MULTICRITÉRIO PELO MÉTODO TODIM
}

\author{
Luiz Flavio Autran Monteiro Gomes* \\ Ibmec/RJ \\ Rio de Janeiro - RJ, Brasil \\ autran@ibmecrj.br \\ Francisco José Coelho Maranhão \\ OGX Oil \& Gas \\ Rio de Janeiro - RJ, Brasil \\ francisco.maranhao@ogx.com.br \\ * Corresponding author / autor para quem as correspondências devem ser encaminhadas \\ Recebido em 08/2007; aceito em 09/2008 após 1 revisão \\ Received August 2007; accepted September 2008 after one revision
}

\section{Resumo}

O problema abordado neste artigo foi o da seleção da melhor opção para destinação das reservas de gás natural descobertas recentemente na Bacia de Santos, mais especificamente no campo de Mexilhão. A análise multicritério empregada no estudo fez uso do método TODIM, método multicritério discreto fundamentado na Teoria dos Prospectos. A aplicação do método TODIM mostrou-se bastante útil na recomendação de opções de projetos no upstream, devido ao fato de se poder identificar claramente as alternativas mais importantes, em face aos cenários testados e em relação aos critérios explicitados. Como complemento ao estudo aqui apresentado, pesquisas relacionadas com o comportamento dos executivos no processo de tomada de decisão, principalmente acerca das questões de motivação e desejos pessoais, poderão também contribuir de maneira decisiva para um melhor entendimento das recomendações e escolhas apontadas.

Palavras-chave: gás natural; decisões multicritério; teoria dos prospectos.

\begin{abstract}
The problem dealt with in this article was the selection of the best option for destination of natural gas reserves recently discovered in the Santos Basin, more specifically in Mexilhão. The particular objectives of the study were: (1) to create a mechanism for supporting the process of analysis and selection of the best options for destination of natural gas; (2) to allow the decision makers choosing among investment opportunities aligned to the expectations and strategies of the investing firm; and (3) facilitate the systemic identification of new investment options. The multicriteria analysis made use of the TODIM method. This is a methods based on Prospect Theory that has proved to be useful in this particular study. The article closes with recommendations for future extensions of this study.
\end{abstract}

Keywords: natural gas; multicriteria decisions; prospect theory. 


\section{Introdução}

O campo de Mexilhão é uma das mais significativas descobertas de gás natural localizada no antigo Bloco BS-400, na Bacia de Santos. O campo está localizado numa posição muito favorável, situado no litoral de São Paulo, a cerca de 165 quilômetros da costa (Figura 1) e a uma lâmina d'água (profundidade entre a superfície e o leito do mar) entre 320 e 550 metros.

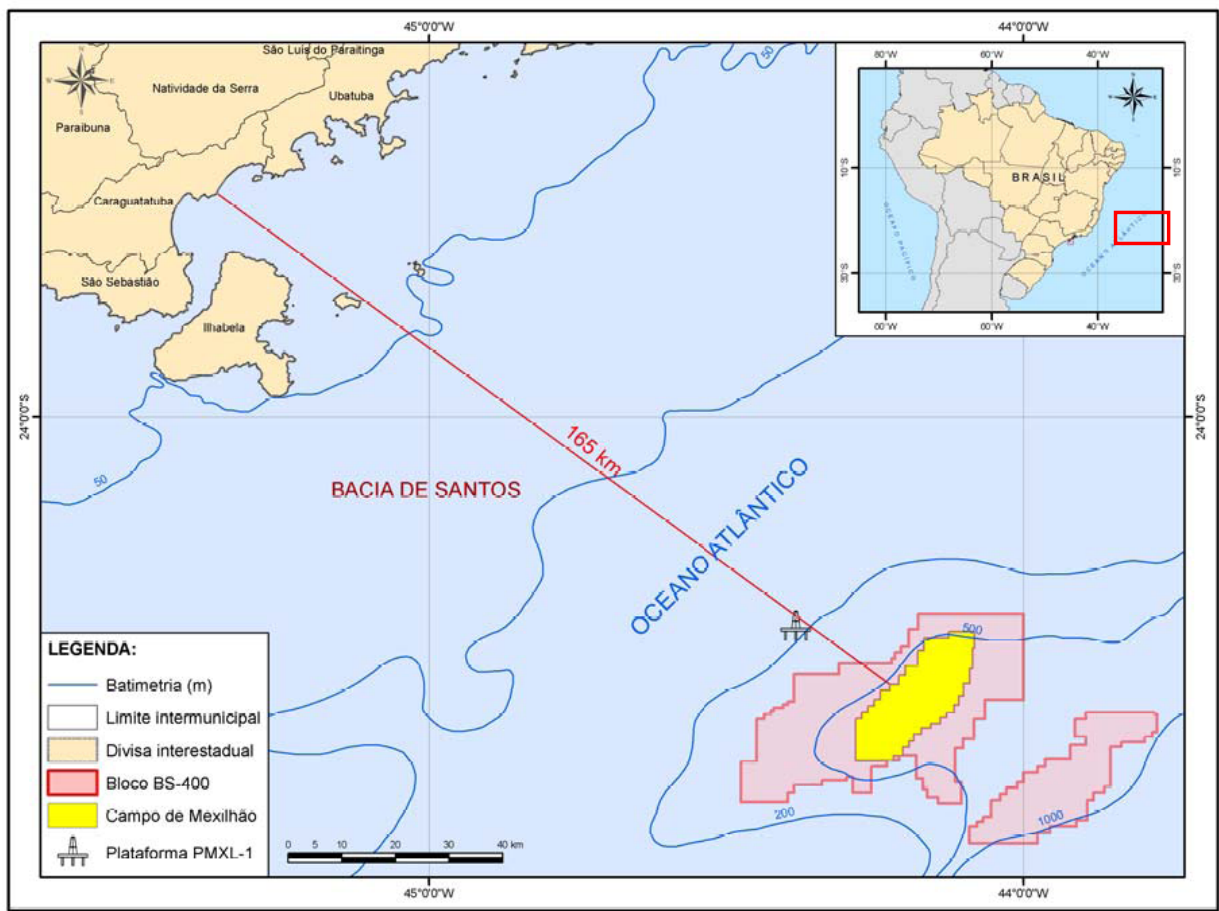

Figura 1 - Mapa com a localização do Campo de Mexilhão.

As reservas de gás natural em Mexilhão são da ordem de 2.52 tcf (trilhões de pés cúbicos), sendo as reservas de óleo associado em cerca de $10 \mathrm{mmbbl}$ (milhões de barris).

Na perfuração do poço 1BRSA-0220 (SPS-36), inicialmente encontrou-se óleo e a Petrobras notificou a ANP desta descoberta em 5 de Agosto de 2003, enquanto o poço ainda estava sendo perfurado.

$\mathrm{Na}$ época da descoberta o mercado doméstico de gás natural não apresentava desenvolvimento ou uma área geográfica de consumo que demandasse esta oferta adicional no curto e médio prazos. Entretanto, diversos acontecimentos contribuem significativamente para o crescimento da demanda de gás natural, tanto no Brasil, quanto na Região da América do Sul. Eventos como o crescimento industrial no Brasil dos últimos anos, potencial 'apagão' de energia (base hídrica) nos próximos anos, crise com a Bolívia e crise energética na Argentina, crescimento da demanda mundial de gás natural direcionado, principalmente, pelos Estados Unidos, além de outros, estão levando à revisão dos planos para destinação de tais reservas. 


\section{Definição do Problema}

O problema abordado neste artigo foi o da seleção da melhor opção para destinação das reservas de gás natural descobertas recentemente na Bacia de Santos, mais especificamente no campo de Mexilhão (BP, 2006).

Embora diversas alternativas possam ser consideradas, do ponto de vista de uma empresa potencialmente investidora o nível de informação sobre as possíveis conseqüências de cada alternativa geralmente deixa muito a desejar e não leva em consideração alguns aspectos fundamentais. Assim, os critérios de decisão tradicionalmente utilizados em um problema desta natureza focam apenas algumas características como, por exemplo: o retorno, o preço, os volumes, o custo e o investimento - aspectos econômico-financeiros, basicamente. Entretanto, não consideram outros critérios igualmente importantes, como: estabilidade política e econômica, ambiente regulatório, regime fiscal, oferta e demanda, dentre outros. Por outro lado, o processo de tomada de decisão deve atender ao grande objetivo segundo o qual, qualquer que seja a opção escolhida, esta deve alavancar melhor a oportunidade, não prejudicando a posição estratégica do decisor - seja este investidor privado ou governo -, sem ignorar outras oportunidades conseqüentes e levando em conta a totalidade de critérios de decisão relevantes.

Os objetivos específicos deste estudo, dados pela empresa, foram: (1) criar um mecanismo que auxilie no processo de análise e seleção das melhores opções de destinação do gás natural recentemente descoberto na Bacia de Santos e, mais especificamente, no campo de Mexilhão (Maranhão et al., 2004); (2) possibilitar aos tomadores de decisão a escolha das opções de investimentos mais alinhadas com as expectativas e objetivos, observadas as estratégias da empresa; e (3) permitir a identificação de forma mais sistemática, clara e abrangente, de novas oportunidades nos mercados onde a empresa atua, ou tenha interesse em atuar, levando em consideração uma base maior e mais diversificada dos principais aspectos que devem influenciar na escolha ou recomendação. O estabelecimento destes objetivos serviu para a identificação do conjunto de critérios de avaliação. Estes serão explicitados mais adiante neste artigo e foram oriundos basicamente de consultas à literatura publicada na área e de consultas a técnicos especialistas do setor, alguns dos quais membros do corpo técnico da própria empresa interessada. Desta forma, aqueles três objetivos específicos foram desdobrados inicialmente em doze critérios de avaliação. Este conjunto inicial de critérios foi reduzido posteriormente, o que conduziu ao conjunto final de oito critérios de avaliação, como se mostrará mais adiante neste artigo.

Note-se que, na indústria do gás, o fator político tem um peso muito grande. Assim, a tomada de decisão de se fazer ou não um investimento, ou mesmo, qual projeto deve ser priorizado em detrimento de outros, é um processo extremamente complexo. Torna-se ainda mais complexo quando quem decide sobre o investimento é uma empresa estrangeira, uma multinacional, nem sempre totalmente familiarizada com os aspectos e peculiaridades locais. Por outro lado, as dificuldades geradas pela tomada de decisão no contexto em pauta não são prerrogativas das companhias e investidores estrangeiros (Johnston, 2003; Real, 2005).

Na próxima seção explica-se o método analítico empregado na atividade de apoio à tomada de decisão para o problema em pauta. 


\section{O Método TODIM}

O método multicritério TODIM (acrônimo de Tomada de Decisão Interativa e Multicritério), concebido e objeto de dois artigos publicados em revista científica européia ao início dos anos 90 (Gomes \& Lima, 1992a; Gomes \& Lima, 1992b), é um método multicritério discreto fundamentado na Teoria dos Prospectos (Kahneman \& Tversky, 1979). Assim sendo, enquanto praticamente todos os demais métodos multicritério partem da premissa de que o tomador de decisão decide buscando sempre a solução correspondente ao máximo de alguma medida global de valor - por exemplo, o maior valor possível de uma função de utilidade multiatributo, no caso da MAUT (Keeney \& Raiffa, 1993; Belton \& Stewart, 2002) —, o método TODIM faz uso da noção de uma medida global de valor calculável pela aplicação do paradigma em que consiste a Teoria dos Prospectos. Com isto, o método se alicerça sobre uma descrição, comprovada por evidências empíricas, de como as pessoas efetivamente decidem em face ao risco (He \& Huang, 2008; Schmidt \& Starmer, 2008).

No que concerne a estruturação do problema de decisão o método TODIM consiste essencialmente em um método multicritério para ordenamento e seleção de alternativas. Como tal, trata-se de um método para avaliar, sob a ótica multicritério, um conjunto de alternativas dadas, não tendo a pretensão de apoiar o decisor no seu posicionamento em face a um determinado contexto. Nesta linha, o método TODIM segue a tradição de autores como Brans \& Mareschal (2002), Roy (1996), Keeney (1992) e Von Winterfeldt \& Edwards (1986). Com tal, pode ser acoplado a um processo de estruturação do problema (Montibeller Neto et al., 2008; Ensslin, Montibeller Neto \& Noronha, 2001; Bana e Costa et al., 1999; Rosenhead, 1989; Belton, Ackermann \& Sheperd, 1997). Por conseguinte, embora a ênfase subjacente ao uso prático do método TODIM esteja na modelagem em si do problema, bem como nos cálculos subseqüentes, reconhece-se a grande necessidade de que se dê a atenção devida a tal processo de estruturação. No caso específico do estudo de caso aqui apresentado, a empresa na qual o estudo foi desenvolvido, através de processo de intensas discussões do qual participaram seus executivos e analistas técnicos, chegou a uma compreensão consensualmente aceita do problema. Esta compreensão caracterizou o que se entende por um modelo requisito para o problema (Phillips, 1984, 1990). A mesma incluiu a explicitação dos objetivos - estes decomponíveis nos critérios de decisão - e a identificação das alternativas viáveis. Esta formulação do problema foi dada pela empresa interessada ao analista de decisão, tendo este optado pelo emprego do método TODIM. Esta escolha do método calcou-se na combinação de sua relativa simplicidade de utilização, viabilizada por meio de uma planilha Excel ${ }^{\mathbb{R}}$, com a originalidade da sua fundamentação na Teoria dos Prospectos, imprimindo assim uma dimensão de praticidade e realismo aos resultados obtidos (Kumar \& Lim, 2008; Huber, Viscusi \& Bell, 2008; Jou et al., 2008).

De modo a poder aplicar o paradigma da Teoria dos Prospectos a uma base de dados proveniente de cálculos e de juízos de valor, o método TODIM deve testar formas específicas das funções de perdas e de ganhos. Estas, uma vez validadas empiricamente, servirão para construir-se a função de diferença aditiva do método, a qual fornece medidas de dominância de cada alternativa sobre cada outra alternativa. Embora pareça complicado terse que testar aquela validade da aplicação do paradigma à base de dados - o que poderia eventualmente obrigar o analista de decisão a usar outras formas das funções de perdas e de ganhos -, na verdade não o é, pois, desde os primeiros usos práticos do método TODIM, publicados na literatura sobre o Apoio Multicritério à Decisão ainda no início dos anos noventa, as mesmas duas formas matemáticas tem sido empregadas com sucesso, tendo sido validadas empiricamente em diferentes aplicações (Rangel \& Gomes, 2007; Gomes \& 
Rangel, 2007; Passos \& Gomes, 2005; Costa, Almeida \& Gomes, 2003; Passos \& Gomes, 2002; Gomes et al., 2001; Trotta, Nobre \& Gomes, 1999; Gomes, Duarte \& Moraes, 1999). Uma apresentação completa da teoria do método TODIM pode ainda ser encontrada em Gomes, Araya \& Carignano, p. 137-157 (2004).

A partir da construção da já mencionada função de diferença aditiva do TODIM - a qual funciona como uma função de valor multiatributo e, como tal, deve ter seu uso também validado, pela verificação da condição de independência preferencial mútua (Keeney \& Raiffa, 1993; Clemen \& Reilly, 2001) - o método conduz a uma ordenação global das alternativas. Observe-se que se constrói a função de valor multiatributo - ou função de diferença aditiva - do método TODIM partindo-se de uma projeção das diferenças entre os valores de duas alternativas quaisquer (percebida em relação a cada critério) sobre um critério referencial ou critério de referência. O conceito de função de diferença aditiva empregado pelo método TODIM baseia-se na pesquisa de Tversky sobre o tratamento analítico da multidimensionalidade de uma função de valor (Tversky, 1969).

O método TODIM faz uso de comparações por pares entre os critérios de decisão, possuindo recursos tecnicamente simples e corretos para eliminar eventuais inconsistências provenientes dessas comparações. Também permite efetuar-se juízos de valor em uma escala verbal, utilizar uma hierarquia de critérios, juízos de valor nebulosos e fazer uso de relações de interdependência entre alternativas (Gomes, Araya \& Carignano, 2004).

Roy \& Bouyssou (1993), sobre o método TODIM, afirmam que este é: “...um método tendo como base a Escola Francesa e a Escola Americana. Combina aspectos provenientes da Teoria de Utilidade Multiatributo, do método AHP e dos métodos ELECTRE” (p. 638).

A idéia, presente na formulação do método TODIM, de introduzir-se na mesma função de valor multiatributo expressões de perdas e de ganhos aufere a esse método alguma similaridade com os métodos PROMÉTHÉE (Brans \& Mareschal, 2002; 1990), que fazem uso da noção de fluxo líquido de superação. Barba-Romero \& Pomerol (2000) compreenderam isto, afirmando o seguinte, a respeito do método TODIM: "baseia-se em uma noção bastante parecida com a de fluxo líquido, no sentido do PROMÉTHÉE” (p. 229).

Considere-se um conjunto de $\mathrm{n}$ alternativas a serem ordenadas na presença de $\mathrm{m}$ critérios quantitativos ou qualitativos, e admita-se que um destes critérios pode ser considerado como o de referência. Após a definição destes elementos, pede-se a especialistas que estimem, para cada um dos critérios qualitativos c, a contribuição de cada alternativa i para o objetivo associado ao critério. Este método requer que os valores das avaliações, das alternativas em relação aos critérios, sejam numéricos e que sejam normalizados. Com isto, os critérios qualitativos avaliados eventualmente numa escala verbal ou nominal são transformados numa escala cardinal. As avaliações dos critérios quantitativos são obtidas através dos desempenhos das alternativas em relação aos critérios, como por exemplo, nível de ruído em decibéis, potência de um motor em HP, nota de um aluno em uma disciplina etc.

Após a avaliação das alternativas em relação a todos os critérios, obtém-se a matriz de avaliação, onde os valores são todos numéricos. Faz-se então a normalização dos mesmos, empregando-se, para cada critério, a divisão do valor de uma alternativa pela soma de todas as alternativas. Essa normalização é feita para cada critério, obtendo-se assim uma matriz, onde todos os valores estão entre zero e um, denominada matriz de desejabilidades parciais $\mathrm{W}=\left[\mathrm{W}_{\mathrm{nm}}\right]$, sendo que $\mathrm{n}$ indica o número de alternativas e $\mathrm{m}$ o número de critérios, conforme se mostra na Tabela 1. Uma vez que a escala onde se lê a medida do desempenho estimado de cada alternativa com respeito a cada critério é determinada a partir do próprio conjunto de

Pesquisa Operacional, v.28, n.3, p.491-509, Setembro a Dezembro de 2008 
alternativas, por meio da normalização supracitada, pode-se minimizar a eventual ocorrência de reversão de ordem pelos dois caminhos seguintes: (i) adição de uma alternativa nova que contribua para expandir o intervalo de variação dos valores normalizados; ou, alternativamente, (ii) ponderação de cada alternativa com relação a um critério com o valor de uma unidade da escala em que o critério é medido (Belton \& Stewart, 2002, p. 159; Belton \& Gear, 1983, 1985; Tallarico, 1990).

Tabela 1 - Matriz de Desejabilidades Parciais.

\begin{tabular}{|c|c|c|c|c|c|c|}
\hline \multirow{2}{*}{ Alternativas } & \multicolumn{5}{|c|}{ Critérios } \\
\cline { 2 - 7 } & $\mathbf{C}_{\mathbf{1}}$ & $\mathbf{C}_{\mathbf{2}}$ & $\ldots$ & $\mathbf{C}_{\mathbf{j}}$ & $\ldots$ & $\mathbf{C}_{\mathbf{m}}$ \\
\hline $\mathrm{A}_{1}$ & $\mathrm{~W}_{11}$ & $\mathrm{~W}_{12}$ & $\ldots$ & $\mathrm{W}_{1 \mathrm{j}}$ & $\ldots$ & $\mathrm{W}_{1 \mathrm{~m}}$ \\
\hline $\mathrm{A}_{2}$ & $\mathrm{~W}_{21}$ & $\mathrm{~W}_{22}$ & $\ldots$ & $\mathrm{W}_{2 \mathrm{j}}$ & $\ldots$ & $\mathrm{W}_{2 \mathrm{~m}}$ \\
\hline$\ldots$ & $\ldots$ & $\ldots$ & $\ldots$ & $\ldots$ & $\ldots$ & \\
\hline $\mathrm{A}_{\mathrm{i}}$ & $\mathrm{W}_{\mathrm{i} 1}$ & $\mathrm{~W}_{\mathrm{i} 2}$ & $\ldots$ & $\mathrm{W}_{\mathrm{ij}}$ & $\ldots$ & $\mathrm{W}_{\mathrm{im}}$ \\
\hline$\ldots$ & $\ldots$ & $\ldots$ & $\ldots$ & & $\ldots$ & $\ldots$ \\
\hline $\mathrm{A}_{\mathrm{n}}$ & $\mathrm{W}_{\mathrm{n} 1}$ & $\mathrm{~W}_{\mathrm{n} 2}$ & $\ldots$ & $\mathrm{W}_{\mathrm{nj}}$ & $\ldots$ & $\mathrm{W}_{\mathrm{nm}}$ \\
\hline
\end{tabular}

Após a atribuição dos pesos dos critérios e de sua normalização, deve-se calcular as matrizes de dominância parciais e a matriz de dominância final. Os tomadores de decisão devem indicar o critério de referência r, que é sempre o critério de maior peso. Assim, $a_{\mathrm{rc}}$ representa a taxa de substituição do critério em análise $\mathrm{c}$ em relação ao critério de referência $\mathrm{r}$. A medida de dominância de cada alternativa i sobre cada alternativa $\mathrm{j}$, incorporada agora à Teoria dos Prospectos, é dada pela expressão matemática (1):

$$
\delta(i, j)=\sum_{c=1}^{m} \Phi_{c}(i, j), \forall(i, j)
$$

onde:

$$
\Phi_{c}(i, j)=\mid \begin{array}{ll}
\sqrt{\frac{a_{r c}\left(W_{i c}-W_{j c}\right)}{\sum_{c=1}^{m} a_{r c}}} & \text { se }\left(W_{i c}-W_{j c}\right)>0, \\
0 & \text { se }\left(W_{i c}-W_{j c}\right)=0, \\
\frac{-1}{\theta} \sqrt{\frac{\sum_{c=1}^{m} a_{r c}\left(W_{j c}-W_{i c}\right)}{a_{r c}}} & \text { se }\left(W_{i c}-W_{j c}\right)<0,
\end{array}
$$

Nas expressões acima tem-se:

$\delta(i, j)$ representa a medida de dominância da alternativa i sobre a alternativa j; m é o número de critérios; $\mathrm{c}$ é um critério qualquer, para $\mathrm{c}=1, \ldots, \mathrm{m}$; $\mathrm{a}_{\mathrm{rc}}$ é a taxa de substituição do critério c pelo critério de referência r; $\mathrm{W}_{\mathrm{ic}}$ e $\mathrm{W}_{\mathrm{jc}}$ são, respectivamente, os pesos das alternativas i e j em relação a c; $\theta$ é o fator de atenuação das perdas. 
A expressão $\Phi_{c}(\mathrm{i}, \mathrm{j})$, representa a parcela de contribuição do critério $\mathrm{c}$ à função $\delta(\mathrm{i}, \mathrm{j})$, quando se compara a alternativa $\mathrm{i}$ com a alternativa $\mathrm{j}$. Caso o valor de $\mathrm{W}_{\mathrm{ic}}-\mathrm{W}_{\mathrm{ic}}$ seja positivo, representará um ganho para a função $\delta(i, j)$ e, portanto, será usada a expressão de $\Phi_{\mathrm{c}}(\mathrm{i}, \mathrm{j})$, correspondente, isto é, à equação (2). Caso $\mathrm{W}_{\mathrm{ic}}-\mathrm{W}_{\mathrm{ic}}$ seja nulo, será atribuído o valor zero para $\Phi_{c}(\mathrm{i}, \mathrm{j})$, aplicando-se a equação (3). $S e \mathrm{~W}_{\mathrm{ic}}-\mathrm{W}_{\mathrm{ic}}$ for negativo, $\Phi_{\mathrm{c}}(\mathrm{i}, \mathrm{j})$ será representado pela equação (4). A construção da função $\Phi_{c}(i, j)$ permite, na verdade, o ajustamento dos dados do problema à função de valor da Teoria dos Prospectos, explicando-se assim a aversão e a propensão ao risco. Tal função tem a forma de "S", representada na Figura 2. Acima do eixo horizontal, considerado como referência desta análise, tem-se uma curva côncava representando os ganhos, e, abaixo do eixo horizontal, tem-se uma curva convexa representando as perdas. A parte côncava reflete a aversão ao risco em face aos ganhos, e a parte convexa, por sua vez, simboliza a propensão ao risco, quando se trata de perdas.

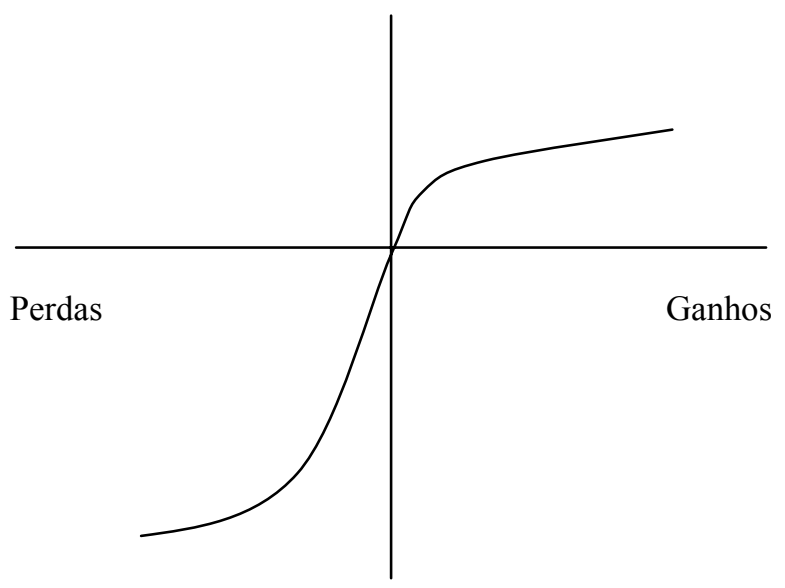

Figura 2 - Função de Valor do Método TODIM.

Depois de calculadas as diversas matrizes de dominância parciais, uma para cada critério, obtém-se a matriz de dominância final $\delta(i, j)$, através da soma dos elementos das diversas matrizes.

A matriz de dominância final é então normalizada, usando-se a expressão (5), para obter o valor global de cada alternativa. Deve-se interpretar cada número calculado como a medida da desejabilidade ou utilidade global, ou, simplesmente, como o valor global de uma alternativa específica. A ordenação das alternativas origina-se da ordenação de seus respectivos valores.

$$
\xi_{i}=\frac{\sum_{j=1}^{n} \delta(i, j)-\min \sum_{j=1}^{n} \delta(i, j)}{\max \sum_{j=1}^{n} \delta(i, j)-\min \sum_{j=1}^{n} \delta(i, j)}
$$

Portanto, o método TODIM determina, a partir das preferências expressas por tomadores de decisão, uma escolha, ao ordenar todas as alternativas. Mudando-se tal conjunto de preferências, pode-se eventualmente chegar um novo resultado, por meio de uma análise de sensibilidade. 


\section{Metodologia do Estudo}

O status quo do processo de tomada de decisão acerca de projetos da empresa, refletindo a prática corrente na mesma, está representado pelo diagrama esquemático apresentado na Figura 3.

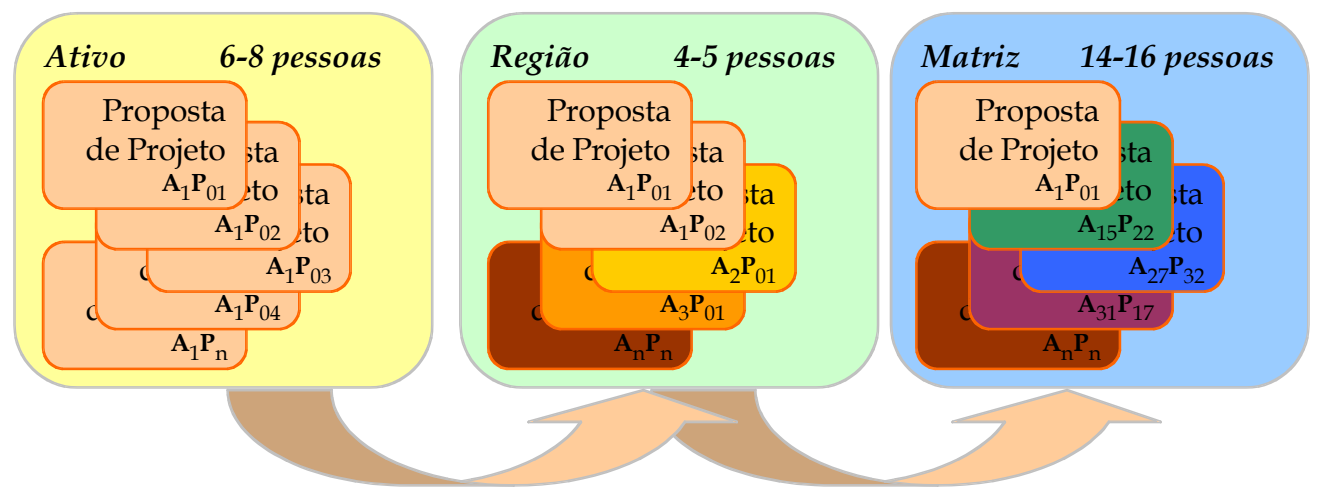

Figura 3 - Modelo esquemático do processo atual de seleção de alternativas.

Nele podemos perceber três níveis no processo decisório, sendo que os dois primeiros têm autonomia para recomendar ou propor alternativas de projetos, que serão considerados na avaliação do portifólio global da empresa, função esta cuja prerrogativa pertence ao terceiro e último nível. É importante notar que os mesmos critérios, de caráter econômico-financeiro, são utilizados de forma consistente em todos os níveis do processo decisório, demonstrando a existência de uma uniformidade quanto à aplicação. Entretanto, os tomadores de decisão também consideram outros aspectos subjetivos de maneira não-uniforme, associando assim um grau de informalidade ao processo de seleção.

Assim, para sua aplicação no processo decisório da empresa, seguiram-se os seguintes passos: (i) levantamento de critérios; (ii) comparação por pares entre os critérios, usando-se para isto a escala de Saaty (1991); (iii) atribuição de uma nota entre 0 e 1 a cada uma das alternativas, relativamente a cada um dos critérios; (iv) análise de sensibilidade; e (v) confronto com o status quo, com tratamento matemático relacionado ao método nas etapas pertinentes. $\mathrm{O}$ passo (ii) produz uma matriz recíproca positiva, com seus elementos no intervalo $[1,9]$, que é usada essencialmente para se obter os pesos dos critérios. Estes pesos são obtidos seguindo-se as seguintes etapas: (1) somam-se os elementos da matriz ao longo de cada coluna e formam-se os recíprocos das somas assim obtidas; (2) divide-se cada recíproco pela soma dos recíprocos, com isto obtendo-se uma primeira listagem dos pesos fazendo isto, ao critério genérico c corresponderá o peso de critério genérico $\mathrm{V}_{0 \mathrm{c}}$. Estas etapas (1) e (2), segundo Saaty (1991, p. 24), consistem na melhor estimativa do autor vetor principal normalizado, correspondente àquela matriz recíproca positiva. Se esta matriz recíproca positiva que produziu o conjunto de pesos de critérios genéricos $V_{0 c}$ não for absolutamente consistente - ou seja, se nela a propriedade da transitividade (Vansnick, 1990, p. 83) não for obedecida -, ainda assim será possível corrigi-la. Para isto, em cada célula da matriz revista, correspondente ao cruzamento do critério c com o critério c', introduz-se o quociente $\mathrm{V}_{0 \mathrm{c}} / \mathrm{V}_{0 \mathrm{c}}$. A nova matriz de comparações por pares entre critérios será então absolutamente consistente, uma vez que nela a propriedade da transitividade estrita é 
respeitada. Esta técnica para se chegar à uma matriz recíproca positiva consistente respeita as comparações paritárias realizadas inicialmente pelo tomador de decisão, sem causar o eventual constrangimento de pedir que o mesmo reveja alguns de seus juízos de valor (Gomes, 1993).

A nota entre 0 e 1 atribuída a cada uma das alternativas, relativamente a cada um dos critérios, pode ser obtida de duas formas distintas. Caso se trate de um critério quantitativo, divide-se a medida - por exemplo, a estimativa do custo financeiro de uma alternativa pela soma dos custos financeiros de todas as alternativas. Caso se trate de um critério qualitativo - como a estimativa da importância social de uma alternativa -, atribui-se a cada destas uma nota entre 1 e 9 e, em seguida, divide-se cada uma dessas notas pela soma das mesmas. Fazendo isto, forma-se a denominada matriz de desejabilidades parciais $\mathrm{W}=\left[\mathrm{W}_{\mathrm{nm}}\right]$, já mencionada anteriormente neste artigo.

As alternativas apresentadas são tratadas como opções por siglas $\mathrm{OP}_{1}, \mathrm{OP}_{2} \ldots \mathrm{OP}_{8}$. Informações acerca destas alternativas acham-se indicadas na Tabela 2 abaixo:

Tabela 2 - Tabela das alternativas apresentadas.

\begin{tabular}{|c|c|}
\hline Alternativas & Comentários \\
\hline $\begin{array}{l}\text { OP }_{1}-\text { ACELERAR } \\
\text { DESENVOLVIMENTO PARA } \\
\text { MERCADO DOMÉSTICO SEM } \\
\text { BOLÍVIA }\end{array}$ & $\begin{array}{l}\text { Trata-se da aceleração no desenvolvimento da reserva, com } \\
\text { destinação exclusiva ao mercado doméstico e sem a } \\
\text { expansão do gasoduto Bolívia-Brasil. }\end{array}$ \\
\hline $\begin{array}{c}\mathrm{OP}_{2}-\text { ACELERAR } \\
\text { DESENVOLVIMENTO PARA } \\
\text { MERCADO DOMÉSTICO COM } \\
\text { BOLÍVIA } \\
\end{array}$ & $\begin{array}{l}\text { Trata-se da aceleração no desenvolvimento da reserva, com } \\
\text { destinação exclusiva ao mercado doméstico e com a } \\
\text { expansão do gasoduto Bolívia-Brasil. }\end{array}$ \\
\hline $\begin{array}{c}\mathrm{OP}_{3}-\text { ACELERAR } \\
\text { DESENVOLVIMENTO PARA } \\
\text { MERCADO DOMÉSTICO E } \\
\text { EXPORTAÇÃO (GNL) COM } \\
\text { BOLÍVIA }\end{array}$ & $\begin{array}{l}\text { Trata-se da aceleração no desenvolvimento da reserva, com } \\
\text { destinação parcial ao mercado doméstico e exportação via } \\
\text { GNL e com a expansão do gasoduto Bolívia-Brasil. }\end{array}$ \\
\hline $\begin{array}{l}\mathrm{OP}_{4}-\text { DESENVOLVIMENTO } \\
\text { NORMAL PARA MERCADO } \\
\text { DOMÉSTICO SEM BOLÍVIA }\end{array}$ & $\begin{array}{l}\text { Trata-se do desenvolvimento normal da reserva, com } \\
\text { destinação exclusiva ao mercado doméstico e sem a } \\
\text { expansão do gasoduto Bolívia-Brasil. }\end{array}$ \\
\hline $\begin{array}{l}\mathrm{OP}_{5}-\text { DESENVOLVIMENTO }_{\text {NORMAL PARA MERCADO }} \\
\text { DOMÉSTICO COM BOLÍVIA }\end{array}$ & $\begin{array}{l}\text { Trata-se do desenvolvimento normal da reserva, com } \\
\text { destinação exclusiva ao mercado doméstico e com a } \\
\text { expansão do gasoduto Bolívia-Brasil. }\end{array}$ \\
\hline $\begin{array}{l}\mathrm{OP}_{6}-\text { DESENVOLVIMENTO } \\
\text { NORMAL PARA MERCADO } \\
\text { DOMÉSTICO E EXPORTAÇÃO } \\
\text { (GNL) COM BOLÍVIA }\end{array}$ & $\begin{array}{l}\text { Trata-se do desenvolvimento normal da reserva, com } \\
\text { destinação parcial ao mercado doméstico e exportação via } \\
\text { GNL e com a expansão do gasoduto Bolívia-Brasil. }\end{array}$ \\
\hline $\begin{array}{c}\mathrm{OP}_{7}-\mathrm{OPÇ} \tilde{A} \mathrm{O} D U M M Y B E S T \\
C A S E\end{array}$ & $\begin{array}{l}\text { Opção fictícia que possui a melhor graduação em cada um } \\
\text { dos critérios interpretados. }\end{array}$ \\
\hline $\begin{array}{c}\mathrm{OP}_{8}-\mathrm{OPÇÃO} \mathrm{DUMMY} \mathrm{WORST} \\
\text { CASE }\end{array}$ & $\begin{array}{c}\text { Opção fictícia que possui a pior graduação em cada um dos } \\
\text { critérios interpretados. }\end{array}$ \\
\hline
\end{tabular}


Os critérios foram levantados junto à literatura publicada na área e por meio de consultas a técnicos do setor, constituindo a lista abaixo: (a) Retorno; (b) Risco; (c) Impactos SócioAmbientais; (d) Tecnologia Disponível; (e) Suprimento; (f) Demanda; (g) Aspecto Político; (h) Alinhamento com Estratégia da Cia.; (i) Regulação Fiscal; (j) Regulação HSSE (Health Safety Security Environment); (k) Regulação de Preço e Mercado; (1) Timing de Implantação.

Com base na técnica de Minimização Heurística da Interdependência entre Critérios, ou MHIC, os mesmos critérios tiveram sua interdependência minimizada, resultando no novo conjunto representado por: (A) Retorno vs. Risco; (B) Impacto Sócio-Ambiental da Alternativa; (C) Tecnologia Disponível; (D) Regulação Geral (fiscal, HSSE, de preço, de mercado); (E) Aspectos Políticos; (F) Alinhamento com Estratégia da Empresa; (G) Balanço Demanda vs. Suprimento Nacional; (H) Timing de Implementação da opção. A técnica MHIC consiste em: (i) separar os critérios por famílias; (ii) analisar sua interdependência internamente em cada família, através de uma matriz; (iii) agrupar os critérios resultantes, não interdependentes, numa matriz, novamente e; (iv) analisá-los finalmente quanto à interdependência. O que a técnica MHIC faz basicamente é, por meio da identificação de interações conceituais entre os doze critérios inicialmente estabelecidos, associar a cada cruzamento destes um nível de força de interação, lido em uma escala verbal pré-definida, de níveis crescentes de interdependência. Isto permite que critérios sejam progressivamente agregados, até que se chegue a um conjunto final de critérios de tamanho mínimo. A heurística em que se constitui a técnica MHIC permite assim a redução da família inicial de critérios (Gomes, Damázio \& Araújo, 1992).

Com base na técnica MHIC reduziu-se o conjunto inicial que compreendia doze critérios. Com isto, chegou-se ao novo conjunto seguinte:

- Retorno vs. Risco: corresponde ao agrupamento dos critérios que compõem esta relação e representa a desejabilidade ideal entre retorno e risco, através de uma escala que parte do mínimo risco e máximo retorno (melhor situação) até máximo risco e mínimo retorno (pior situação).

- Impacto Sócio-Ambiental da Alternativa: corresponde ao grau de impacto provocado na natureza e na sociedade. Representa a expectativa do que pode ser provocado pela aceitação, continuidade, ou descontinuidade da alternativa através de uma escala que tem em si os impactos: (i) negativo; (ii) nenhum e; (iii) positivo.

- Tecnologia Disponível: corresponde à tecnologia disponível para consecução da alternativa e representa seu impacto empresarial através de uma escala que parte de baixo custo e disponibilidade abundante até a inexistência de tecnologia.

- Regulação (fiscal, HSSE, de preço, de mercado): corresponde ao amadurecimento para o setor nos seus diversos aspectos e representa sua importância através de uma escala que passa da inexistência de regulação, a parcial e a regulação madura.

- Aspectos Políticos: corresponde à influência dos Aspectos Políticos na implementação da alternativa, representada através de uma escala que vai de influência negativa à influência positiva, passando uma escala de influência nula (ou sem influência).

- Alinhamento com Estratégia da Empresa: corresponde ao grau de alinhamento da alternativa com a estratégia empresarial e representa a expectativa de valor agregado de um projeto a esta estratégia, através de uma escala que parte de fracamente alinhado a fortemente alinhado, sendo este o mais desejável. 
- Balanço Demanda vs. Suprimento Nacional: corresponde ao grau de alinhamento da opção proposta com a atual projeção do balanço entre a demanda e o suprimento de gás natural no país, representado através de uma escala que possui quatro níveis de graduação: (i) Baixo Alinhamento, (ii) Médio Alinhamento, (iii) Bom Alinhamento e (iv) Alto Alinhamento.

- Timing de Implementação da opção: corresponde ao tempo necessário para efetiva implementação da alternativa, representado por uma escala que vai desde relativamente rápido até muito demorado, passando por tolerável.

Ainda após essa redução, é desejável que os tomadores de decisão sejam confrontados com o conjunto resultante de critérios, com vistas a se testar a potencial validade funcional da mesma. Isto permite que esses tenham um envolvimento ativo na modelagem do problema, aqui incluída, e, com o devido destaque, a obtenção da lista final de critérios de avaliação. Para tanto, validou-se uma planilha em Excel $^{\circledR}$ entre os tomadores de decisão, em seus diversos níveis, relacionados à decisão em questão. Buscou-se sempre, nas interações com esses tomadores de decisão, a maior simplicidade possível no manuseio e compreensão da terminologia. A planilha foi distribuída eletronicamente a 20 tomadores de decisão da empresa, sendo composta por 3 colunas e 28 linhas. As colunas das extremidades continham os critérios a serem comparados par a par, resultando em 28 comparações, usando-se a escala de Saaty (1991), apenas no aspecto verbal. Procedeu-se à passagem dos valores lidos nas escalas verbais ou nominais à escala de 0 até 1 por meio da associação da leitura 0 ao pior valor possível e da leitura 100 ao melhor valor possível. A partir daí, solicitou-se aos tomadores de decisão que posicionassem cada leitura naquelas escalas dentro do intervalo $[0,1]$. Os valores dos pesos normalizados dos critérios foram então determinados, usando-se para isto o procedimento de cálculo explicado anteriormente neste artigo. A Tabela 3 apresenta a média dos pesos dos critérios depois da normalização.

Tabela 3 - Pesos médios dos critérios.

\begin{tabular}{|c|c|}
\hline Critérios & Médias \\
\hline Relação Retorno vs. Risco & 0,3273 \\
\hline Impactos Sócio-Ambientais & 0,1910 \\
\hline Balanço Demanda vs. Suprimento nacional & 0,1473 \\
\hline Regulação (fiscal, HSSE, preço, mercado) & 0,1104 \\
\hline Aspecto Político & 0,0782 \\
\hline Alinhamento com Estratégia da Cia. & 0,0670 \\
\hline Tecnologia disponível & 0,0508 \\
\hline Timing de implantação da opção & 0,0280 \\
\hline
\end{tabular}

\section{Resultados da Aplicação do Método TODIM}

A atribuição de pesos aos critérios, feita pelo decisor, gera a matriz de desejabilidades, que está apresentada na Tabela 4.

O gráfico da Figura 4 demonstra nitidamente o resultado deste experimento aplicando dois fatores de atenuação a perdas $(\theta=1$ e $\theta=5)$, ambos com correlação igual. Nessa Figura, o valor $\theta=1$ corresponde ao segmento da função de valor mais próximo do semi-eixo negativo 
das ordenadas, ao passo que $\theta=5$ corresponde ao segmento daquela função de valor que está mais próximo do semi-eixo negativo das abscissas. Nesses dois casos, uma regressão estatística indicou que tanto faz obter-se a ordenação final fazendo-se $\theta=1$ ou $\theta=5$, uma vez que se chega ao mesmo coeficiente de regressão, de $\mathrm{R}^{2}=0,8425$, quando se introduz cada um desses dois valores na equação (4). Esse parâmetro $\theta$ funciona como um fator de atenuação das perdas: alterações no valor de $\theta$ poderão eventualmente conduzir a resultados diferentes, em termos da ordenação final (Gomes \& Rangel, 2007). Nesta aplicação em particular observou-se que, sendo os dois valores $\theta=1$ e $\theta=5$ igualmente aceitáveis - sob o aspecto de ajustamento das nuvens de pontos localizados no quadrante negativo por meio da equação (4) à forma clássica da função de valor da Teoria dos Prospectos (Kahneman \& Tversky, 1979) - obtém-se a mesma ordenação final, conforme indicado na Tabela 5. Em essência, em aplicações do método TODIM é sempre desejável que se faça uma análise da sensibilidade dos resultados a variações em $\theta$ e, no caso de se obter ordenações finais diferentes, será a interação entre o analista e os tomadores de decisão que determinará qual valor de $\theta$ será empregado nos cálculos. Pode-se perceber ainda que, para graus de aversão diferentes, apesar do aumento da aversão, as preferências são praticamente mantidas, e a ordenação das opções também não foi alterada, como pode ser verificado na Tabela 4. Como se mencionou anteriormente neste artigo, a desejabilidade de cada alternativa indica seu valor global. A ordenação das alternativas origina-se da ordenação de seus respectivos valores.

Somente com a alteração do $\theta=10$, houve uma quase imperceptível mudança: inversão de posições entre a $\mathrm{OP}_{5}$ e $\mathrm{OP}_{2}$. Por meio de análises de sensibilidade observa-se que houve um reposicionamento entre as $\mathrm{OP}_{5}$ e $\mathrm{OP}_{2}$, o que ratifica o teste de sensibilidade preliminar realizado em torno da variação de $\theta$. No segundo teste, alterando somente os três critérios mais importantes, não houve qualquer alteração no quadro de ordenação das opções.

Tabela 4 - Desejabilidades das alternativas conforme os critérios.

\begin{tabular}{|c|c|c|c|c|c|c|c|c|}
\hline \multicolumn{7}{|c|}{ Matriz de Desejabilidades } \\
\hline Critérios & \multicolumn{7}{|c|}{ Opç̃̃es } \\
\cline { 2 - 9 } & $\mathbf{O P}_{\mathbf{1}}$ & $\mathbf{O P}_{\mathbf{2}}$ & $\mathbf{O P}_{\mathbf{3}}$ & $\mathbf{O P}_{\mathbf{4}}$ & $\mathbf{O P}_{\mathbf{5}}$ & $\mathbf{O P}_{\mathbf{6}}$ & $\mathbf{O P}_{\mathbf{7}}$ & $\mathbf{O P}_{\mathbf{8}}$ \\
\hline Relação Retorno vs. Risco & 4,0 & 3,0 & 3,0 & 4,0 & 3,0 & 3,0 & 4,0 & 1.0 \\
\hline Impactos Sócio-Ambientais & 4,0 & 3,0 & 3,0 & 3,0 & 2,0 & 2,0 & 4,0 & 1,0 \\
\hline $\begin{array}{c}\text { Balanço Demanda vs. } \\
\text { Suprimento nacional }\end{array}$ & 4,0 & 2,0 & 3,0 & 2,0 & 3,0 & 3,0 & 4,0 & 1,0 \\
\hline $\begin{array}{c}\text { Regulação (fiscal, HSSE, } \\
\text { preço, mercado) }\end{array}$ & 2,0 & 2.0 & 2,0 & 2,0 & 2,0 & 2,0 & 3,0 & 1,0 \\
\hline Aspecto Político & 3,0 & 1,0 & 1,0 & 3,0 & 1,0 & 3,0 & 3,0 & 1,0 \\
\hline $\begin{array}{c}\text { Alinhamento com Estratégia } \\
\text { da Empresa }\end{array}$ & 2,0 & 2,0 & 1,0 & 2,0 & 2,0 & 2,0 & 3,0 & 1,0 \\
\hline Tecnologia Disponível & 3,0 & 3,0 & 2,0 & 3,0 & 3,0 & 3,0 & 4,0 & 1,0 \\
\hline $\begin{array}{c}\text { Timing de Implementação da } \\
\text { Opção }\end{array}$ & 3,0 & 1,0 & 2,0 & 3,0 & 1,0 & 3,0 & 1,0 & 3,0 \\
\hline
\end{tabular}

Resultados para valor $\theta=1$ 


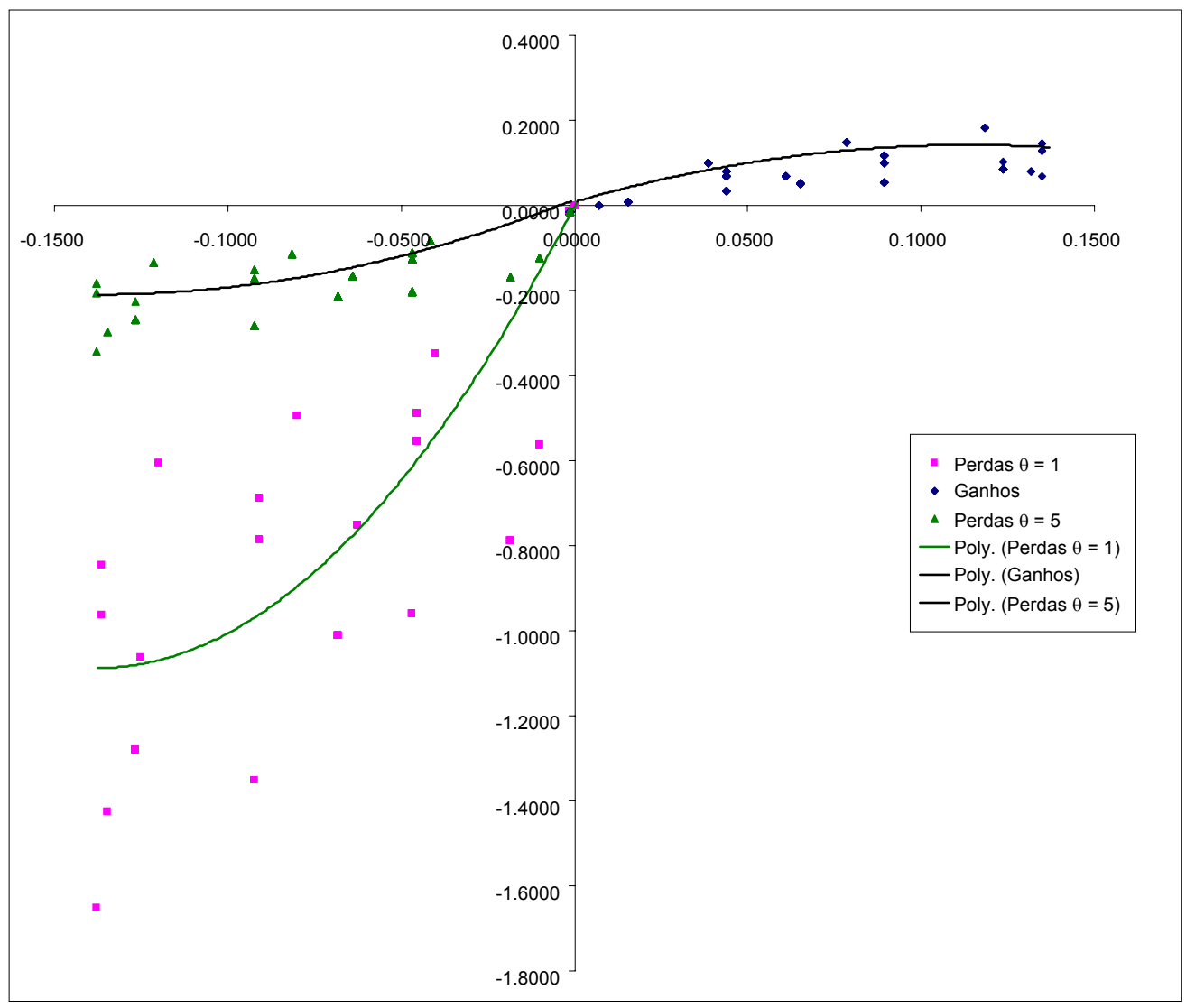

Figura 4 - Gráfico de Perdas e Ganhos utilizando $\theta=5$ e $\theta=1$.

Tabela 5 - Resultado da ordenação com a variação de $\theta$.

\begin{tabular}{|c|c|c|c|c|c|c|}
\hline \multirow{3}{*}{ Ordenações } & \multicolumn{3}{|c|}{$\theta=1$} & \multicolumn{3}{|c|}{$\theta=5$} \\
\hline & \multirow{2}{*}{$\begin{array}{l}\text { Alterna- } \\
\text { tivas }\end{array}$} & \multicolumn{2}{|c|}{ Desempenhos } & \multirow{2}{*}{$\begin{array}{l}\text { Alterna- } \\
\text { tivas }\end{array}$} & \multicolumn{2}{|c|}{ Desempenhos } \\
\hline & & Brutos & $\begin{array}{l}\text { Normali- } \\
\text { zados }\end{array}$ & & Brutos & $\begin{array}{c}\text { Normali- } \\
\text { zados }\end{array}$ \\
\hline $1^{\mathrm{o}}$ & $\mathrm{OP}_{7}$ & 4,0071 & 1,0000 & $\mathrm{OP}_{7}$ & 4,0071 & 1,0000 \\
\hline $2^{o}$ & $\mathrm{OP}_{1}$ & $-2,8623$ & 0,8542 & $\mathrm{OP}_{1}$ & 1,5933 & 0,8088 \\
\hline $3^{\circ}$ & $\mathrm{OP}_{4}$ & $-7,9990$ & 0,7451 & $\mathrm{OP}_{4}$ & $-0,1725$ & 0,6690 \\
\hline $4^{\mathrm{o}}$ & $\mathrm{OP}_{6}$ & $-9,3098$ & 0,7173 & $\mathrm{OP}_{6}$ & $-0,8519$ & 0,6152 \\
\hline $5^{\circ}$ & $\mathrm{OP}_{5}$ & $-11,7867$ & 0,6647 & $\mathrm{OP}_{5}$ & $-1,5820$ & 0,5574 \\
\hline $6^{\circ}$ & $\mathrm{OP}_{2}$ & $-12,0187$ & 0,6598 & $\mathrm{OP}_{2}$ & $-1,6065$ & 0,5554 \\
\hline $7^{\circ}$ & $\mathrm{OP}_{3}$ & $-22,1036$ & 0,4457 & $\mathrm{OP}_{3}$ & $-3,6593$ & 0,3929 \\
\hline $8^{\circ}$ & $\mathrm{OP}_{8}$ & $-43,1017$ & 0,0000 & $\mathrm{OP}_{8}$ & $-8,6203$ & 0,0000 \\
\hline
\end{tabular}


A Tabela 5, por sua vez, apresenta um quadro comparativo da ordenação obtida pela aplicação do método TODIM $(\theta=1)$, em confronto com a ordenação resultante da aplicação de metodologia de avaliação clássica (combinação focada em fatores econômicofinanceiros), utilizada atualmente pela empresa, denominada status quo. É importante mencionar que as informações obtidas junto à empresa consideram somente critérios técnicos de avaliação. Questões ligadas aos aspectos objetivos e/ou subjetivos não padronizados, tais como preferências por parte dos tomadores de decisão, ligação com outros projetos em andamento, restrições globais de recursos, concorrência de projetos no portfólio etc, não foram levadas em consideração na elaboração da ordenação do status quo.

Tabela 6 - Demonstrativo dos Resultados da ordenação método TODIM vs. status quo.

\begin{tabular}{|c|c|c|l|}
\hline Ordenações & TODIM & Status quo & \multicolumn{1}{|c|}{ Comentários Preliminares } \\
\hline $1^{\circ}$ & $\mathrm{OP}_{1}$ & $\mathrm{OP}_{3}$ & $\begin{array}{l}\text { Oposição total na comparação entre o resultado dos } \\
\text { métodos. }\end{array}$ \\
\hline $2^{\mathrm{o}}$ & $\mathrm{OP}_{4}$ & $\mathrm{OP}_{6}$ & $\begin{array}{l}\text { Ganho relativo de posição na comparação do } \\
\text { resultado dos métodos. }\end{array}$ \\
\hline $3^{\mathrm{o}}$ & $\mathrm{OP}_{6}$ & $\mathrm{OP}_{1}$ & $\begin{array}{l}\text { Perda significativa de posição na comparação do } \\
\text { resultado dos métodos. }\end{array}$ \\
\hline $4^{\mathrm{o}}$ & $\mathrm{OP}_{5}$ & $\mathrm{OP}_{5}$ & $\begin{array}{l}\text { Posição mantida independente da aplicação dos } \\
\text { métodos. }\end{array}$ \\
\hline $5^{\mathrm{o}}$ & $\mathrm{OP}_{2}$ & $\mathrm{OP}_{2}$ & $\begin{array}{l}\text { Posição mantida independente da aplicação dos } \\
\text { métodos. }\end{array}$ \\
\hline $6^{\mathrm{o}}$ & $\mathrm{OP}_{3}$ & $\mathrm{OP}_{4}$ & $\begin{array}{l}\text { Oposição total na comparação entre o resultado dos } \\
\text { métodos. }\end{array}$ \\
\hline
\end{tabular}

Mesmo sem acesso às premissas e informações adicionais que nortearam a ordenação das opções, resultantes da aplicação da metodologia clássica (status quo), presume-se que o grande peso de fatores econômico-financeiros teve impacto significativo no posicionamento invertido, comparativamente ao método TODIM, das opções $\mathrm{OP}_{3}(\boldsymbol{\uparrow})$ e $\mathrm{OP}_{4}(\downarrow)$.

\section{Limitações do Estudo}

A aplicação do método TODIM mostrou-se bastante útil na recomendação de opções de projetos no upstream, devido ao fato de podermos identificar as alternativas mais importantes por meio do cálculo multicritério, vis-a-vis os cenários testados e em relação aos critérios explicitados. Deve, no entanto, ficar bem claro que a aplicação do método TODIM pressupõe uma ampla estruturação do problema. No caso particular deste estudo, tal estruturação, a rigor, não foi feita, uma vez que a empresa preferiu chegar a uma compreensão consensual do problema por meio de intensas discussões entre seus executivos e técnicos. Reconhece-se que esta é uma foi uma limitação deste estudo de caso, sendo fundamental que, em estudos semelhantes futuros, a empresa dê atenção devida ao processo de estruturação do problema, o que será definitivamente enriquecedor sob todos os aspectos.

Não houve neste estudo a preocupação de se buscar comparar o resultado ao qual se chegou pelo método TODIM com aquele que seria obtido com algum outro método multicritério. 
A razão disto foi que a intenção subjacente ao estudo aqui apresentado era mostrar a empresa que a utilização de um método multicritério poderia esclarecer aspectos do processo não usualmente abordados pela metodologia corriqueiramente em uso na mesma, designada aqui pela expressão status quo. Este esclarecimento conduziu efetivamente a uma percepção, pela empresa, de que um elenco mais amplo tanto de alternativas como de oportunidades de decisão poderia emergir se aplicações sistemáticas do apoio multicritério à decisão fossem feitas, antecedidas de uma adequada estruturação do problema (Ensslin, Montibeller \& Noronha, 2001). Neste sentido, considera-se que aquela intenção foi plenamente atendida por meio deste estudo.

Seria uma presunção assumir que haja um método de apoio à decisão perfeito, independente das particularidades do caso estudado. Para cada tipo de decisão pode haver um ou mais métodos igualmente bons que, mediante seu entendimento e uso adequado, promovam ajustes para o alinhamento aos paradigmas que o alicerçam, além do ambiente de negócios e da qualidade das informações, permitam uma aplicação consistente com resultados bem suportados. A combinação de dois ou mais métodos pode atuar tanto na função de complementariedade, quanto do refino dos resultados, por prover filtros consecutivos.

Uma das limitações que ocorreu durante a execução deste estudo diz respeito à acessibilidade. Não foi possível o acesso a todos os tomadores de decisão durante a coleta de dados via questionários e entrevistas de validação. Entretanto, o nível de colaboradores respondentes (18 entre 20 acessíveis), dentre executivos e técnicos da empresa, permitiu uma massa crítica bastante significativa para o bom desenvolvimento do estudo.

O sigilo sobre as premissas acerca das alternativas, ordenadas mediante a aplicação da metodologia clássica utilizada atualmente na empresa, aqui denominada status quo, foi preservado por questões de sensibilidade de informações estratégicas da organização. Tal restrição prejudica parcialmente o estudo, na medida em que não é possível uma comparação detalhada das ordenações obtidas pela aplicação de ambos os métodos, bem como a identificação das razões para as discrepâncias significativas.

A modelagem de cenários de decisões cotidianas, como compras e suprimentos, mostrou-se relativamente restrita, visto que tais cenários são passíveis de implementação de políticas de decisões, minimizando o efeito dos prospectos, provocado pela aversão a perdas, induzindo, portanto, a aplicação de um método diferente.

\section{Conclusão e Sugestões para Estudos Futuros}

Em estudos futuros a estruturação do problema deve definitivamente anteceder ao emprego do método multicritério, agregando inestimável valor à aplicação deste. Outra conclusão deste estudo diz respeito ao amadurecimento do processo de tomada de decisão, mediante o aprofundamento da modelagem. Em função do detalhamento aplicado, é possível a identificação de eventuais inversões na ordenação das opções, o que permitiria a reavaliação destas, em função de restrições de recursos, por exemplo.

Questões ligadas a disparidades de juízos de valor, bem como a multiplicidade de variáveis envolvidas, são demandadas no processo decisório, o que coloca os tomadores de decisão em posição não muito confortável e, por vezes, conflitante com os interesses particulares de cada tomador de decisão e da empresa. Qual a opção mais racional para empresa? Os argumentos de defesa das alternativas escolhidas são realmente conclusivos e inquestionáveis? Estas são

Pesquisa Operacional, v.28, n.3, p.491-509, Setembro a Dezembro de 2008 
perguntas que pairam quando da finalização de um ciclo de planejamento ou de uma seleção eventual. O processo de decisão corrente na empresa demanda um tempo considerado relativamente grande na compilação de dados e geração de informações, bem como nas diversas rodadas de discussão das recomendações. A metodologia do estudo aqui apresentada, alicerçada no uso do método multicritério TODIM, contribuiu para a elaboração de um plano racional de contingência da empresa, permitindo a análise de mudanças de cenários, sem necessariamente gerar-se o recálculo de dados e a produção de novas informações.

O confronto dos resultados da ordenação dos projetos, apresentados pela aplicação do método TODIM, não objetiva a invalidação do processo atual. Todavia, mostra que é possível minimizar o esforço de priorização e dedicar tempo à análise de sensibilidade em diversas dimensões, uma questão de extrema importância para a indústria de petróleo e gás natural. Ainda com relação ao futuro de pesquisas na área do Apoio Multicritério à de Decisão, o método TODIM se apresenta como um método multicritério para o qual se pode esperar uma boa aceitação em função da sua fundamentação teórica, constituída pela Teoria dos Prospectos, pela oportunidade de seu enfoque interativo, e, sem dúvida, pela praticidade na sua aplicação.

O estudo aqui apresentado, no entanto, não se encerra com esta conclusão, nem os resultados podem ser considerados plenos ou definitivos. Ao contrário, muitos aspectos ligados as Teorias da Decisão e dos Prospectos requerem maior aprofundamento e estudo, visando sua aplicação no ambiente empresarial da indústria de petróleo e gás natural. Como complemento ao estudo aqui apresentado, estudos adicionais relacionadas com atitudes e comportamentos dos executivos e técnicos especialistas no processo de tomada de decisão, principalmente acerca das questões de motivação, vieses e estilos pessoais de participação, poderão contribuir de maneira decisiva para um melhor esclarecimento do processo decisório, conduzindo, portanto, a recomendações e escolhas potencialmente melhores (Hammond, Kenney \& Raiffa, 1999; Nutt, 2002).

\section{Agradecimentos}

Os autores agradecem a revisores anônimos pelos comentários e críticas referentes à primeira versão deste artigo. Tais comentários possibilitaram que se melhorasse substancialmente aquela primeira versão.

\section{Referências Bibliográficas}

(1) Bana e Costa, C.A.; Ensslin, L.; Corrêa, E.C. \& Vansnick, J. (1999). Decision support systems in action: integrated application in a multicriteria decision AID process. European Journal of Operational Research, 113, 315-335.

(2) Barba-Romero, S. \& Pomerol, J.C. (2000). Multicriterion Decision in Management: principles and practice. Kluwer Academic Publishers, Boston.

(3) Belton, V.; Ackermann, F. \& Sheperd, I. (1997). Integrated support for problem structuring through an alternative evaluation using COPE and V.I.S.A. Journal of Multi-Criteria Decision Analysis, 6, 115-130.

(4) Belton, V. \& Gear, T. (1983). On a short-coming of Saaty's method of analytic hierarchies. Omega, 11(3), 228-230. 
(5) Belton, V. \& Gear, T. (1985). The legitimacy of rank reversal. Omega, 13(3), 143-144.

(6) Belton, V. \& Stewart, T.J. (2002). Multiple criteria decision analysis: an integrated approach. Kluwer Academic Publishers, Massachusetts.

(7) BP (2006). Statistical Review of World Energy 2005. Disponível em: $<$ http://www.bp.com>.

(8) Brans J.-P. \& Mareschal, B. (1990). The PROMÉTHÉE methods for MCDM, the PROMCALC GAIA and BANDADVISER software. In: Readings in Multiple Criteria Decision Aid [edited by C.A. Bana e Costa], chapter 2. Springer Verlag, Berlin.

(9) Brans, J.-P. \& Mareschal, B. (2002). Prométhée-Gaia Une Méthodologie d'Aide à la Décision en Présence de Critères Multiples. Université de Bruxelles/Ellipses, Bruxelles.

(10) Clemen, R.T. \& Reilly, T. (2001). Making hard decisions with decision tools ${ }^{\circledR}$. Duxbury, Pacific Grove.

(11) Costa, A.P.C.S.; Almeida, A.T. de \& Gomes, L.F.A.M. (2003). Priorização do Portfolio de Projetos de Sistemas de Informação Baseado no Método TODIM de Apoio Multicritério à Decisão. Investigación Operativa, vol. XI, 23, 106-119.

(12) Ensslin, L.; Montibeller Neto, G. \& Noronha, S.M.D. (2001). Apoio à Decisão: Metodologias para Estruturação de Problemas e Avaliação Multicritério de Alternativas. $1^{\mathrm{a}}$ ed. Insular, Florianópolis.

(13) Gomes, L.F.A.M. (1993). Efficient Reduction of Inconsistency in Pairwise Comparison Metrices. Systems Analysis Modelling Simulation, 11(4), 333-335.

(14) Gomes, L.F.A.M.; Araya, M.C.G. \& Carignano, C. (2004). Tomada de Decisões em Cenários Complexos. Pioneira Thomson, Rio de Janeiro.

(15) Gomes, L.F.A.M.; Damázio. H.N. \& Araújo, G.M. de (1992). Minimização heurística da interdependência entre critérios no auxílio multicritério à decisão - Uma aplicação à decisão sobre seguro ambiental para transporte rodoviário de produtos perigosos. Working paper, Departamento de Engenharia Industrial, PUC-Rio, Rio de Janeiro, outubro.

(16) Gomes, L.F.A.M.; Duarte, V.C.A. \& Moraes, L.F.R. (1999) Análise Multicritério de Projetos de Produção de Petróleo: Os Métodos PROMETHÉE e TODIM. Pesquisa Naval, 1999(12), 251-262.

(17) Gomes, L.F.A.M.; Duarte, V.C.A.; Silva, C.F. \& Hanszmann, S. (2001). Um Enfoque Multicritério à Priorização de Projetos Tecnológicos. Investigación Operativa, 20, 41-54.

(18) Gomes, L.F.A.M. \& Lima, M.M.P.P. (1992a). TODIM: basics and application to multicriteria ranking oof projects with environmental impacts. Foundations of Computing and Decision Sciences, 16(4), 113-127.

(19) Gomes, L.F.A.M. \& Lima, M.M.P.P. (1992b). From modeling individual preferences to multicriteria ranking of discrete alternatives: a look at Prospect Theory and the additive difference model. Foundations of Computing and Decision Sciences, 17(3), 171-184. 
(20) Gomes, L.F.A.M. \& Rangel, L.A.D. (2007). An Application of the TODIM Method to the Multicriteria Rental Evaluation of Residential Properties. Aceito para publicação no European Journal of Operational Research, disponível on-line em $<\mathrm{http} / / /$ www.sciencedirect.com>, doi: 10.1016/j.ejor.2007.10.046.

(21) Hammond, J.S.; Keeney, R.L. \& Raiffa, H. (1999). Smart Choices - A practical guide to making better decisions. Harvard Business School Press, Boston.

(22) He, Y. \& Huang, R.-H. (2008). Risk attributes theory: decision making under risk. European Journal of Operational Research, 186(1), 243-260, April.

(23) Huber, J.; Viscusi, W.K. \& Bell, J. (2008). Reference dependence in interactive choices. Organizational Behavior \& Human Decision Processes, 106(2), 143-152, July.

(24) Johnston, D. (2003). International Exploration Economics, Risk and Contract Analysis. PennWell Corporation, Tulsa.

(25) Jou, R.-C.; Kitamura, R.; Weng, M.-C.W. \& Chen, C.-C.C. (2008). Dynamic commuter departure time choice under uncertainty. Transportation Research Part A: Policy \& Practice, 42(5), 774-783, June.

(26) Kahneman, D. \& Tversky, A. (1979). Prospect theory: An analysis of decision under risk. Econometrica, 47, 263-292.

(27) Kenney, R.L. (1992). Value-Focused Thinking: A Path to Creative Decision Making. Harvard University Press, Cambridge.

(28) Keeney, R.L. \& Raiffa, H. (1993). Decisions with multiple objectives: preferences and value tradeoffs. Cambridge University Press, Cambridge.

(29) Kumar, A. \& Lim, S.S. (2008). How do decision frames influence the stock investment choices of individual investors? Management Science, 54(6), 1052-1064, June.

(30) Maranhão, F.J.C.; Teixeira, M.; Sivieri, R. \& Bacellar, R.M.H. de (2004). Estudo de Viabilidade de Produção e Exportação de Gás Natural Liquefeito (GNL) da Bacia de Santos para os EUA. Projeto Final de Pós-Graduação, COPPEAD/UFRJ, Rio de Janeiro.

(31) Montibeller Neto, G.; Belton, V.; Ackermann, F. \& Ensslin, L. (2008). Reasoning maps for decision aid: an integrated approach for problem-structuring and multi-criteria evaluation. Journal of the Operational Research Society, 59, 575-589.

(32) Nutt. P.C. (2002). Why Decisions Fail Avoiding the blunders and traps that lead to debacles. Berrett-Koehler Publishers, San Francisco.

(33) Passos, A.C. \& Gomes, L.F.A.M. (2005). Enfoque Multicritério à Teoria das Prospectivas. Revista de Administração Mackenzie, 6(1), 60-77.

(34) Passos, A.C.; Gomes, L.F.A.M. (2002). Análise para Escolha de Material de Emprego Militar: Um Enfoque Multicritério. Pesquisa Naval, 15, 273-289.

(35) Phillips, L.D. (1984). A theory of requisite decision models. Acta Psychologica, 52, $29-48$.

(36) Phillips, L.D. (1990). Requisite decision modelling for technological projects. In: Social Decision Methodology for Technological Projects [edited by C. Vlek \& G. Cvetkovich], Kluwer Academic Publishers, Dordrecht, 95-110. 
(37) Rangel, L.A.D. \& Gomes, L.F.A.M. (2007). Determinação do valor de referência do aluguel de imóveis residenciais empregando o método TODIM. Pesquisa Operacional, 27(2), 357-372.

(38) Real, R.V. (2005). Fatores Condicionantes ao Desenvolvimento de Projeto de GNL para o Cone Sul: uma alternativa para a monetização das reservas de gás da região. Dissertação de Mestrado, COPPE/UFRJ, Rio de Janeiro.

(39) Rosenhead, J. (ed.) (1989). Rational Analysis for a Problematic World. John Wiley \& Sons, Chichester.

(40) Roy, B. (1996). Multicriteria Methodology for Decision Aiding. Kluwer Academic Publishers, Dordrecht.

(41) Roy, B. \& Bouyssou, D. (1993). Aide Multicritère à la Décision: méthodes et cas. Economica, Paris.

(42) Saaty, T.L. (1991). Método de Análise Hierárquica. Makron Books, São Paulo.

(43) Schmidt, U. \& Starmer, C. (2008). Third-generation prospect theory. Journal of Risk \& Uncertainty, 36(3), 203-223, June.

(44) Tallarico, M.C. da F. (1990). Reversão de Ordem em Alguns Métodos Multicriteriais de Decisão. Dissertação de Mestrado em Engenharia de Produção, Departamento de Engenharia Industrial, PUC-Rio, Rio de Janeiro.

(45) Tversky, A. (1969). Intransitivity of preferences. Psychological Review, 76(1), 31-48.

(46) Trotta, L.T.F. \& Nobre, F.F. \& Gomes, L.F.A.M. (1999). Multi-Criteria Decision Making - an approach to setting priorities in health care. Statistics in Medicine, 18, 3345-3354.

(47) Vansnick, J.-C. (1990). Measurement theory and decision aid. In: Readings in Multiple Criteria Decision Aid [edited by C.A. Bana e Costa], Springer-Verlag, Berlin, 81-100.

(48) Von Winterfeldt, D. \& Edwards, W. (1986). Decision Analysis and Behavioral Research. Cambridge University Press, Cambridge. 